\title{
Pegylated G-CSF Inhibits Blood Cell Depletion, Increases Platelets, Blocks Splenomegaly, and Improves Survival after Whole-Body Ionizing Irradiation but Not after Irradiation Combined with Burn
}

\author{
Juliann G. Kiang, ${ }^{1,2,3}$ Min Zhai, ${ }^{1}$ Pei-Jyun Liao, ${ }^{1}$ David L. Bolduc, ${ }^{1}$ \\ Thomas B. Elliott, ${ }^{1}$ and Nikolai V. Gorbunov ${ }^{1}$ \\ ${ }^{1}$ Radiation Combined Injury Program, Armed Forces Radiobiology Research Institute, Bethesda, MD 20889, USA \\ ${ }^{2}$ Department of Radiation Biology, Uniformed Services University of the Health Sciences, Bethesda, MD 20814, USA \\ ${ }^{3}$ Department of Medicine, Uniformed Services University of the Health Sciences, Bethesda, MD 20814, USA
}

Correspondence should be addressed to Juliann G. Kiang; juliann.kiang@usuhs.edu

Received 18 November 2013; Accepted 22 January 2014; Published 5 March 2014

Academic Editor: Pranela Rameshwar

Copyright $\odot 2014$ Juliann G. Kiang et al. This is an open access article distributed under the Creative Commons Attribution License, which permits unrestricted use, distribution, and reproduction in any medium, provided the original work is properly cited.

\begin{abstract}
Exposure to ionizing radiation alone (radiation injury, RI) or combined with traumatic tissue injury (radiation combined injury, CI) is a crucial life-threatening factor in nuclear and radiological accidents. As demonstrated in animal models, CI results in greater mortality than RI. In our laboratory, we found that B6D2F1/J female mice exposed to ${ }^{60} \mathrm{Co}-\gamma$-photon radiation followed by $15 \%$ total-body-surface-area skin burns experienced an increment of $18 \%$ higher mortality over a 30-day observation period compared to irradiation alone; that was accompanied by severe cytopenia, thrombopenia, erythropenia, and anemia. At the 30th day after injury, neutrophils, lymphocytes, and platelets still remained very low in surviving RI and CI mice. In contrast, their RBC, hemoglobin, and hematocrit were similar to basal levels. Comparing CI and RI mice, only RI induced splenomegaly. Both RI and CI resulted in bone marrow cell depletion. It was observed that only the RI mice treated with pegylated G-CSF after RI resulted in 100\% survival over the 30-day period, and pegylated G-CSF mitigated RI-induced body-weight loss and depletion of WBC and platelets. Peg-G-CSF treatment sustained RBC balance, hemoglobin levels, and hematocrits and inhibited splenomegaly after RI. The results suggest that pegylated G-CSF effectively sustained animal survival by mitigating radiation-induced cytopenia, thrombopenia, erythropenia, and anemia.
\end{abstract}

\section{Introduction}

Injuries induced by ionizing radiation alone (RI) or in combination with trauma from blast and thermal energy exposure $(\mathrm{CI})$ are expected after the detonation of radiation dispersal devices or nuclear weapons. In vivo [1] and in vitro $[2,3]$ studies indicate that RI induced DNA doublestrand breaks (DSBs), activated signal transduction pathways, elevated cytokine/chemokine concentrations in the peripheral blood, and increased systemic bacterial infection, thereby leading to cell death and multiple-organ dysfunction and failure $[1,4-6]$. Traumatic injury followed by RI (i.e., CI) enhanced histopathological responses to RI, thereby increasing the mortality $[1,5-7]$. Because the responses to RI and CI occur at molecular, cellular, tissue, and system levels, the complexity of the responses makes it difficult to identify countermeasures for prophylaxis, mitigation, or therapy.

$\mathrm{RI}$ and CI remarkably increased granulocyte colony stimulating factor (G-CSF) in mouse blood for more than 7 days [7]. The increase was initially believed to be a self-defensive response, but its appearance was probably too late to participate in repairing bone marrow damage. Bone marrow injury usually occurred within hours after RI $[1,2]$. With this consideration, G-CSF and its modified form, that is, pegylated G-CSF (peg-G-CSF), have been used clinically to treat radiation-injured patients [8]. It is reported that this 
growth factor decreased the period of neutropenia or aplasia in the limited number of radiation accident victims studied and also enhanced neutrophil recovery following anticancer therapy [8]. The cytokine activates or primes neutrophils to enhance their function [9]. The peg-G-CSF formulation has a much longer biological half-life than G-CSF [10], thus avoiding the necessity of daily injections, which are deleterious in irradiated mice. The drug has no toxic or adverse effects in mice at the doses used. Akin to G-CSF peptide, peg-G-CSF initiates proliferation and differentiation of myeloid progenitors into mature granulocytes and induces hematopoietic stem cell mobilization from the bone marrow into the bloodstream. It is involved in recovery from infection $[11,12]$ and wound healing [13]. Peg-G-CSF, when combined with stem cell factor and erythropoietin, was successfully used in saving a hospital technician who had accidentally entered a ${ }^{60} \mathrm{Co}$-irradiation therapy room and received a 4.5 Gy dose of radiation [14].

This report, which is intended to stimulate interest in advancing research on peg-G-CSF in support of approval for treatment of radiation-induced neutropenia or aplasia by U.S. Food and Drug Administration, provides data from an experimental animal model designed to demonstrate the efficacy of peg-G-CSF as an effective radiomitigator.

\section{Materials and Methods}

Research was conducted in a facility accredited by the Association for Assessment and Accreditation of Laboratory Animal Care International (AAALACI). All procedures involving animals were reviewed and approved by the AFRRI Institutional Animal Care and Use Committee. Euthanasia was carried out in accordance with the recommendations and guidance of the American Veterinary Medical Association $[15,16]$

2.1. Animals. B6D2F1/J female mice (The Jackson Laboratory, Bar Harbor, ME) were maintained in a facility accredited by the Association for Assessment and Accreditation of Laboratory Animal Care International in plastic microisolator cages on hardwood chip bedding. Commercial rodent chow and acidified tap water were provided ad libitum at 12 to 20 weeks of age. Animal holding rooms were maintained at $21^{\circ} \mathrm{C} \pm 1^{\circ} \mathrm{C}$ with $50 \% \pm 10 \%$ relative humidity using at least 10 changes $/ \mathrm{h}$ of $100 \%$ conditioned fresh air. A 12-h 0600 (light) to 1800 (dark) full-spectrum lighting cycle was used. The AFRRI Institutional Animal Care and Use Committee approved all animal procedures.

2.2. Gamma Irradiation. Mice were given 9.5 Gy [1] wholebody bilateral ${ }^{60} \mathrm{Co}$ gamma-photon radiation, delivered at a dose rate of $0.4 \mathrm{~Gy} / \mathrm{min}$, while held in vertically stacked, ventilated, four-compartment, and acrylic plastic boxes that provided electron equilibrium during irradiation. Empty compartments within the boxes were filled with 3-inchlong, 1-inch-diameter acrylic phantoms to ensure uniform electron scattering. The mapping of the radiation field was performed with alanine/EPR dosimetry [17] using standard alanine calibration sets from NIST and National Physical Laboratory of UK. The mapping provided dose rates to water in the core of the acrylic phantom ( 3 inches long, 1 inch in diameter) in each compartment of the mouse rack on the day of the mapping. The field was uniform within $\pm 1.8 \%$ over all of the 120 compartments. The exposure time for each irradiation was determined from the mapping data; corrections for the ${ }^{60} \mathrm{Co}$ decay and the small differences in the mass energy absorption coefficients for water and soft tissue were applied. The accuracy of the actual dose delivery was verified with an ionization chamber adjacent to the mouse rack, which had been calibrated in terms of dose to soft tissue in the cores of mice.

2.3. Skin Injury. Skin surface injuries were performed on the shaved dorsal surface of mice. Animals receiving skin burns were anesthetized by methoxyflurane inhalation. A $15 \%$ total-body-surface-area skin burn was performed within $1 \mathrm{~h}$ after irradiation using a $1 \times 1$-in custom designed template positioned centrally over the shaved dorsal skin surface. Mice received a 12 -s burn from ignited $95 \%$ ethanol $(0.25 \mathrm{~mL}$, $[5,6])$. All mice subjected to the skin injury were given $0.5 \mathrm{~mL}$ sterile $0.9 \% \mathrm{NaCl}$ intraperitoneally (i.p.), which contained $150 \mathrm{mg} / \mathrm{kg}$ of acetaminophen (AmerisourceBergen, Glen Allen, VA) and $0.05 \mathrm{mg} / \mathrm{kg}$ of buprenorphine immediately after skin injury to alleviate pain. Four hours later, mice were given a second dose of $150 \mathrm{mg} / \mathrm{kg}$ of acetaminophen. For animals receiving skin wounds, a 15\% total body-surfacearea skin wound was performed within $1 \mathrm{~h}$ after irradiation $[5,6]$. Skin-wounded mice received one dose of $150 \mathrm{mg} / \mathrm{kg}$ of acetaminophen immediately after skin injury.

2.4. Pegylated G-CSF. Peg-G-CSF (Neulasta; NDC: 555-13019001) is a polyethylene glycol pharmaceutical-formulatedgrade drug, also known as pegfilgrastim, and was purchased from AmerisourceBergen Corporation (Valley Forge, PA). A dose of $1000 \mu \mathrm{g} / \mathrm{kg}$ was administered by s.c. injection $[18,19]$ in a volume of $0.2 \mathrm{~mL} 24 \mathrm{~h}, 8 \mathrm{~d}$, and $15 \mathrm{~d}$ after RI or CI, that is, $25 \mu \mathrm{g} / 25$-g mouse. Neulasta is supplied in $0.6 \mathrm{~mL}$ prefilled syringes for subcutaneous injection. Each syringe contains $6 \mathrm{mg}$ Peg-G-CSF in a sterile, clear, colorless, and preservativefree solution containing $0.35 \mathrm{mg}$ acetate, $0.02 \mathrm{mg}$ polysorbate 20, $0.02 \mathrm{mg}$ sodium, and $30 \mathrm{mg}$ sorbitol in water for injection, USP. Peg-G-CSF was studied in mice with sham-operation, burns, radiation, or radiation combined with burns.

2.5. G-CSF. G-CSF (Neupogen; Amgen, Inc., Thousand Oaks, CA, NDC: $555-13-546-10)$. A dose of $10 \mu \mathrm{g} / \mathrm{kg}$ was injected s.c. [20] in a volume of $0.2 \mathrm{~mL}$ on day 1 at $24 \mathrm{~h}$ and thereafter once daily on days $2-14$ after RI or CI. The vehicle given to control mice was sterile $0.9 \%$ sodium chloride solution for injection, USP. G-CSF was studied in mice with shamoperation, wounds, radiation, or radiation combined with wounds.

2.6. Antimicrobial Agents. Gentamicin sulfate cream, $0.1 \%$ (generic, E. Fougera and Co., Melville, NY, NDC 0168-00715), was applied daily for 10 days to the skin injuries on days 
1-10. Levofloxacin (LVX) (generic, Aurobindo Pharma, Ltd., Mahaboob Nagar, India, NDC 65862-537-50), $100 \mathrm{mg} / \mathrm{kg}$ in $0.2 \mathrm{~mL} / \mathrm{mouse}$, was administered p.o. daily for 14 days on days 3-16. Briefly, a 500-mg tablet was crushed by mortar and pestle. The LVX in the powder was dissolved in a volume of sterile water approximately one-third the total volume required to prepare the concentration needed for the average body mass of the mice to be treated. The suspension was centrifuged to remove the particulate filler and the supernatant solution was passed through a $0.45-\mu \mathrm{m}$ membrane filter into a sterile amber bottle, which was sealed with a sterile rubber stopper.

2.7. Survival and Body Weight. Animals were monitored at least twice daily for their general health and survival for 30 days. Their body weights were measured on days $0,1,3,7,14$, 21 , and 28 .

\subsection{Assessment of Blood Cell Profile in Peripheral Blood.} Blood samples were collected in EDTA tubes at day 30 after RI or CI and assessed with the ADVIA 2120 Hematology System (Siemens, Deerfield, IL). Differential analysis was conducted using the peroxidase method and the light scattering techniques recommended by the manufacturer.

2.9. Measurements of Spleen Weights and Splenocytes. Spleens were collected from each euthanized mouse at day 30 after RI or CI. Each specimen was weighed and then homogenized in a cell strainer (BD Falcon, Bedford, MA) with 1X Hank's Balanced Salt Solution (Invitrogen, Grand Island, NY). Splenocytes in the buffer were washed with 1X ACK lysis buffer (Invitrogen) to lyse RBC, mixed by vortexing, and centrifuged at $800 \times \mathrm{g}$. Splenocytes were collected and counted using a hemocytometer.

2.10. Measurements of Bone Marrow Cells. Bone marrow cells from femurs were collected at day 30 after RI or CI and washed with $10 \mathrm{~mL} 1 \mathrm{X}$ phosphate-buffered saline (PBS). The cells were then centrifuged at $800 \times \mathrm{g}$, resuspended in $10 \mathrm{~mL}$ $1 \mathrm{X}$ PBS buffer, and then counted using a hemocytometer.

2.11. Statistical Analysis. Parametric data are expressed as the mean \pm s.e.m. For each survival experiment, 20-22 mice per group were tested on an individual basis. Survival analyses were performed using the log-rank test. For cell analysis, oneway ANOVA, two-way ANOVA, studentized-range test, and Student's $t$-test were used for comparison of groups, with $5 \%$ as a significant level.

\section{Results}

3.1. Survival and Body Weight. Skin burn (15\% total-bodysurface area) alone did not result in mortality over a 30day observation period (Figure $1(\mathrm{~d})$ ). However, skin burn following irradiation increased mortality to $50 \%$, which was greater than mortality observed in RI mice (32\%), as shown in Figure 1(a). In RI mice, vehicle treatment did not affect the radiation-induced mortality (Figures $1(\mathrm{~b})$ and 1(d)). Treatment with peg-G-CSF, however, enhanced 30-day survival to $100 \%$ (Figure $1(\mathrm{~b}) ; P=0.0033$ ). In CI mice, both vehicle treatment and peg-G-CSF did not change the CIinduced mortality (Figures 1(c) and 1(d)).

It is evident that RI reduced the body weight [1]. Skin burn alone did not induce body-weight loss but did enhance the radiation-induced body-weight loss (Figure 2(a)). Peg-GCSF treatment reduced the body-weight loss in the RI mice (Figure 2(b)) but did not change body weight in the CI mice (Figure 2(c)).

In a separate protocol, skin wound was performed following irradiation. In this experiment, G-CSF was administered s.c. daily beginning on day 1 at $24 \mathrm{~h}$ and thereafter once daily on days 2-14. As shown in Figure 3, RI and CI control mice, which were given daily injections of vehicle for 14 days, displayed $0 \%$ and $5 \%$ survival, respectively, during the 30 day experimental period. In comparison, survival rates in RI and CI mice given G-CSF were $25 \%(P=0.0001)$ and $20 \%$ $(P=0.0053)$, respectively. All nonirradiated mice survived, which were given G-CSF.

The requirement for repeated daily injection of vehicle or recombinant G-CSF for efficacy added stress to the irradiated mice, which might cause high mortality in this model of combined injury, Once-a-week administration of vehicle or peg-G-CSF reduced the total number of injections per mouse from 14 to 3 , thus alleviating stress to the mice. Therefore, subsequent studies were focused on the effects of peg-G-CSF on mice receiving irradiation or in combination with burns.

3.2. Blood Cell Profile in Peripheral Blood. RI is known to deplete WBC and RBC [1]. Skin burn alone did not affect WBC (Figure 4) but slightly decreased RBC (Figure 5) profiles. In RI mice, peg-G-CSF treatment mitigated WBC depletion slightly (Figure 4(a)), mainly numbers of neutrophils (Figure 4(b)), lymphocytes (Figure 4(c)), and monocytes (Figure $4(\mathrm{~d})$ ) but not in CI mice. This treatment also mitigated decreased RBC numbers (Figure 5(a)), hemoglobin (Figure 5(b)), hematocrit (Figure 5(c)), and platelets (Figure 5(d)) in RI mice.

3.3. Spleen Weight and Splenocytes. In contrast to the effects of CI, RI alone has been shown to significantly increase spleen weight (i.e., splenomegaly) in surviving animals. Skin burn alone did not alter spleen weights and the number of splenocytes (Figure 6). Peg-G-CSF treatment increased the number of splenocytes and spleen weights in sham and burned mice but fully inhibited radiation-induced increases in spleen weight in RI mice (Figure 6(a)) and splenocyte counts below control levels (Figure 6(b)). Treatment with peg-G-CSF also decreased splenocyte counts in CI mice below control levels (Figure 6(b)).

3.4. Bone Marrow Cells. In sham-irradiated mice, vehicle alone did not change the basal level of bone marrow cells; peg-G-CSF treatment, however, significantly elevated bone marrow cell counts (Figure 7). In skin-burned mice, neither the vehicle nor the drug treatment altered the basal level of bone marrow cell counts. Also skin burn did not change cellularity. However, in RI mice, irradiation significantly 


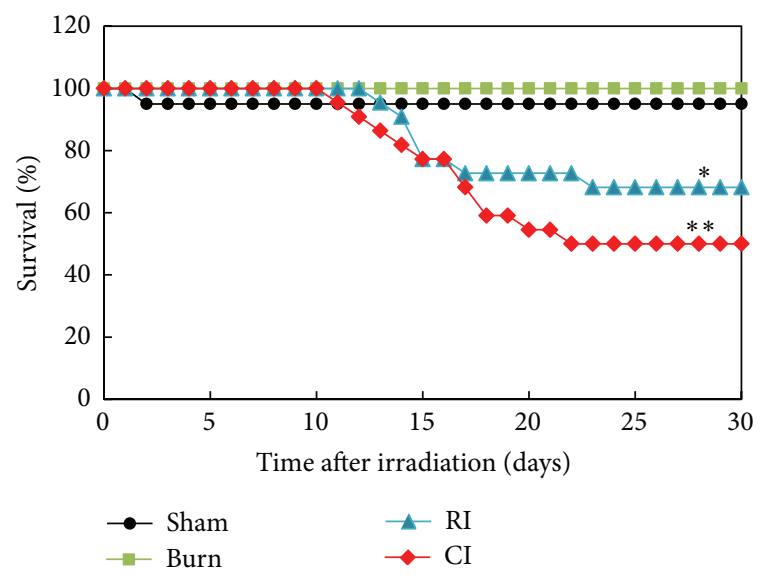

(a) Control mice

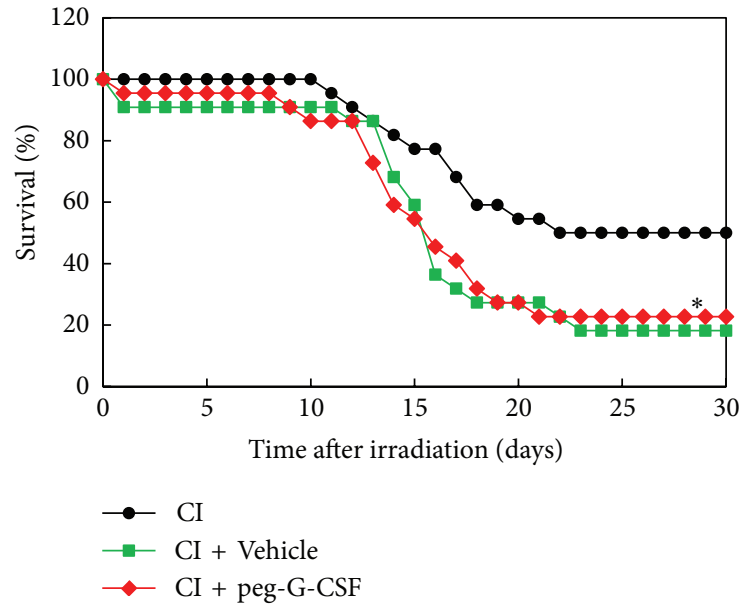

(c) CI mice

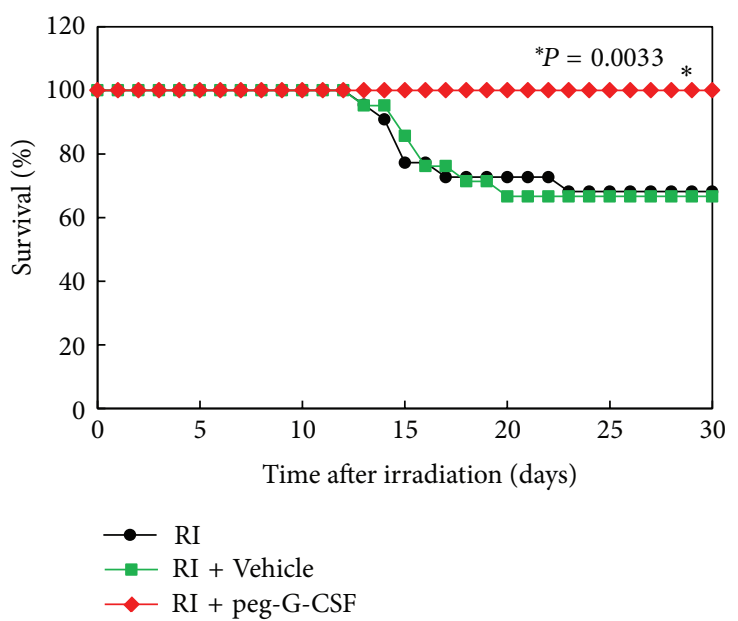

(b) RI mice

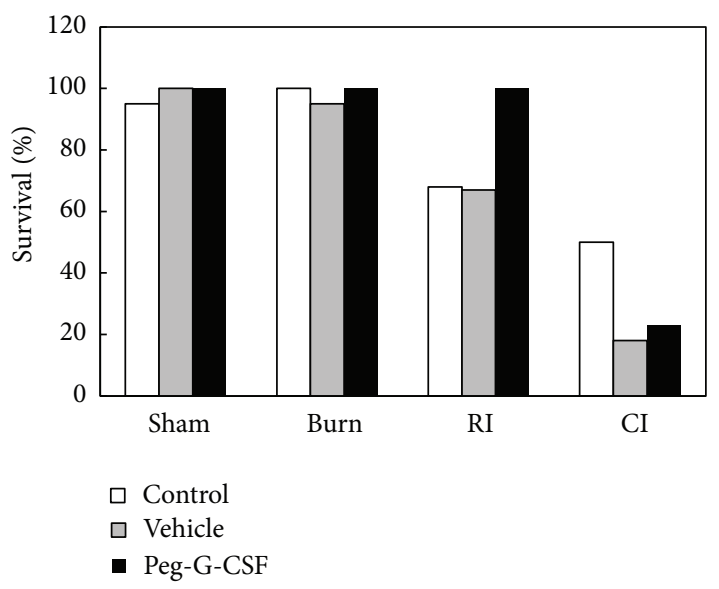

(d) Vehicle versus peg-G-CSF

Figure 1: Peg-G-CSF improved survival after whole-body ionizing irradiation alone but not after irradiation combined with skin burn. $N=20-22$ per group. For (a) ${ }^{*} P<0.05$ versus sham, burn, and CI; ${ }^{* *} P<0.05$ versus sham burn and CI. For (b) ${ }^{*} P=0.0033$ RI + peg-GCSF versus RI + Vehicle and RI. For (c) ${ }^{*} P<0.05$ versus CI. For (d) representing $100 \%$ survival in peg-G-CSF treated RI mice. RI: 9.5 Gy; CI: 9.5 Gy and skin burn.

decreased the bone marrow cell count, whereas both the vehicle treatment and the drug treatment increased the cell counts, but there was no statistical difference between the vehicle treatment and the drug treatment. In CI-mice, pegG-CSF treatment failed to improve the cellularity (Figure 7).

\section{Discussion}

This report presents data that skin burn significantly increased radiation-induced mortality and body-weight loss. The latter was thought due to injured small intestines [7]. However, the skin burn was less potent than skin wound in producing a synergistic effect with radiation in B6D2F1/J mice. These results are consistent with previous observations in rat [21, 22], guinea pig [23], dog [24], swine [25], and mice $[1,5,6,26-29]$. Consequences of either RI or CI include acute myelosuppression, immune system inhibition, fluid imbalance, macro/microcirculation failure, massive cellular damage, and disruption of vital organ functions, which lead to multiple-organ dysfunction syndrome (MODS) and multiple-organ failure (MOF), the most frequent causes of death after irradiation [30-32].

Peg-G-CSF at the dose used displayed 100\% survival in sham-operated and burned mice. Peg-G-CSF treatment enhanced 30 -day survival to $100 \%$ and diminished bodyweight losses after RI; this, however, was not observed in CI mice. We reported that RI and CI induced increases in G-CSF concentrations in serum (on the order of $100-1,000 \mathrm{pg} / \mathrm{mL}$ in RI mice and $2,000-10,000 \mathrm{pg} / \mathrm{mL}$ in CI mice [7]. These increases are important for recovery from RI $[29,33,34]$. In comparison, a dose of peg-G-CSF at $25 \mu \mathrm{g} /$ mouse would yield a maximum serum concentration on the order of $1,000 \mathrm{pg} / \mathrm{mL}$ [35]. Peg-G-CSF has a longer biological half-life than G-CSF [10]. Therefore, daily injections are not necessary, which would be detrimental in irradiated mice. In contrast to therapy with peg-G-CSF, our laboratory has found that 


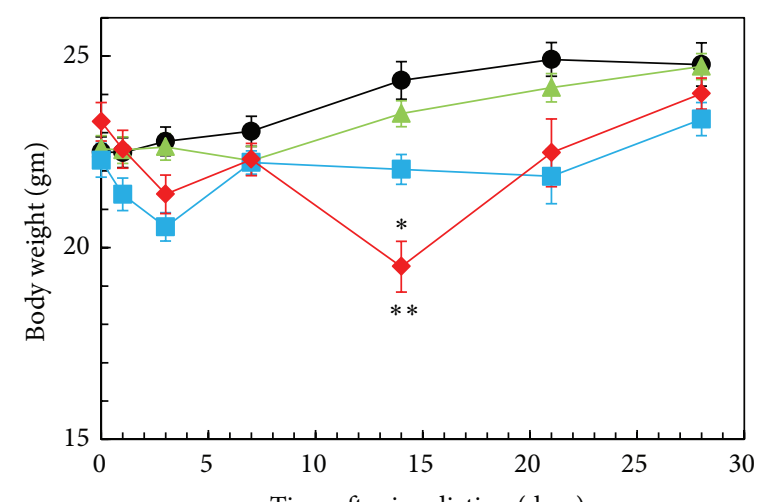

Time after irradiation (days)

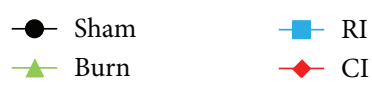

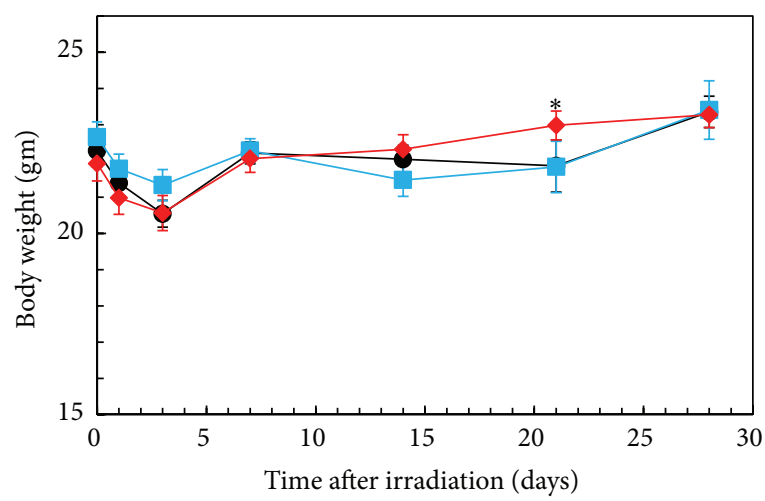

- RI

$-\mathrm{RI}+$ Vehicle

$\neg \mathrm{RI}+$ peg-G-CSF

(a) Control mice

(b) RI mice

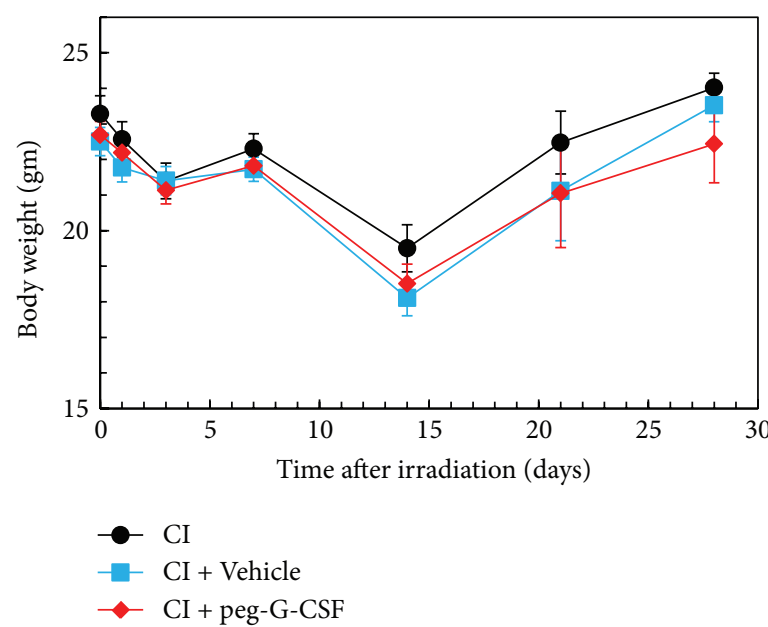

(c) CI mice

Figure 2: Peg-G-CSF significantly improved body-weight loss after whole-body ionizing irradiation alone but not after irradiation combined with skin burn. $N=20-22$ per group. For (a) ${ }^{*} P<0.05$ versus sham, burn, and $\mathrm{CI} ;{ }^{* *} P<0.05$ versus sham, burn, and RI. For (b) ${ }^{*} P<0.05$ versus RI and RI + Vehicle. RI: 9.5 Gy; CI: 9.5 Gy and skin burn.

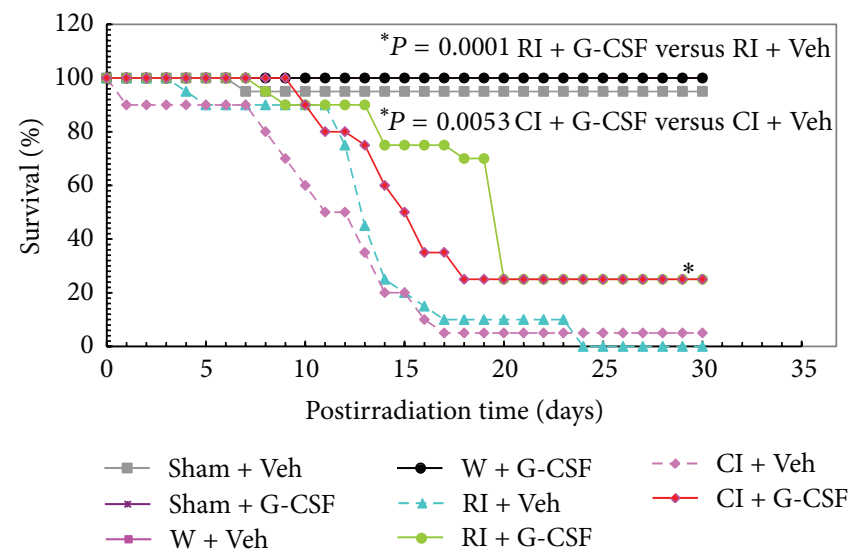

FIGURE 3: G-CSF improved survival after whole-body ionizing irradiation alone or after irradiation combined with skin wound. G-CSF was administered s.c. on day 1 at $24 \mathrm{~h}$ and thereafter once daily on days $2-14$ after RI or CI. $N=20$ per group. ${ }^{*} P=0.0001 \mathrm{RI}+\mathrm{G}-\mathrm{CSF}$ versus RI + Veh; ${ }^{*} P=0.0053$ CI + G-CSF versus CI + Veh. Veh: vehicle; RI: 9.5 Gy; CI: 9.5 Gy and skin wound. 


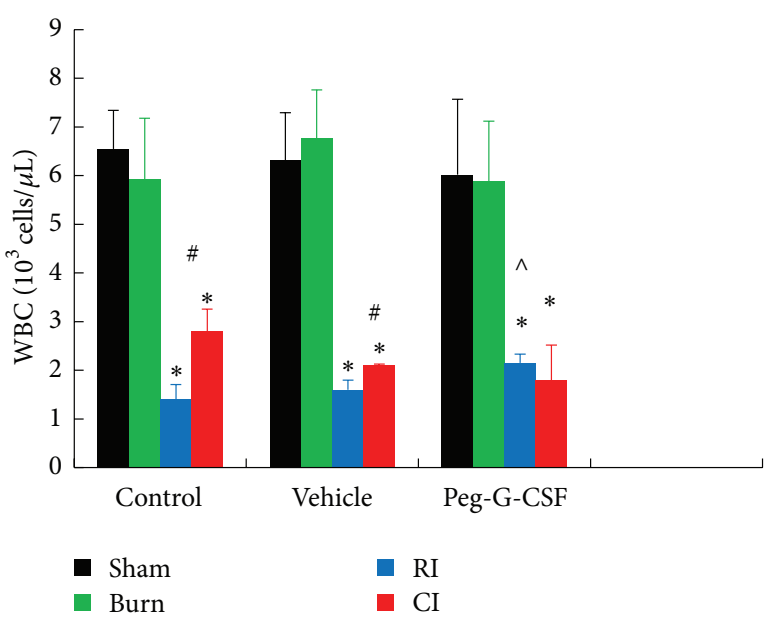

(a) WBC

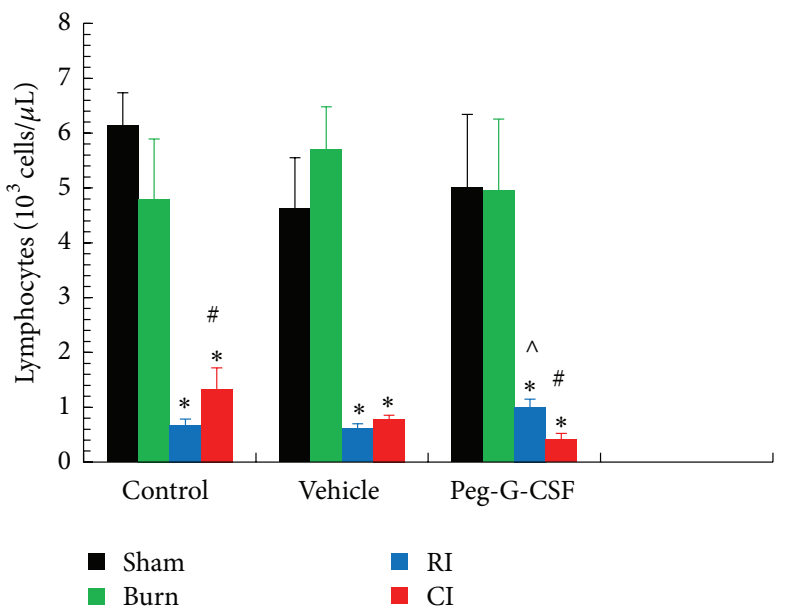

(c) Lymphocytes

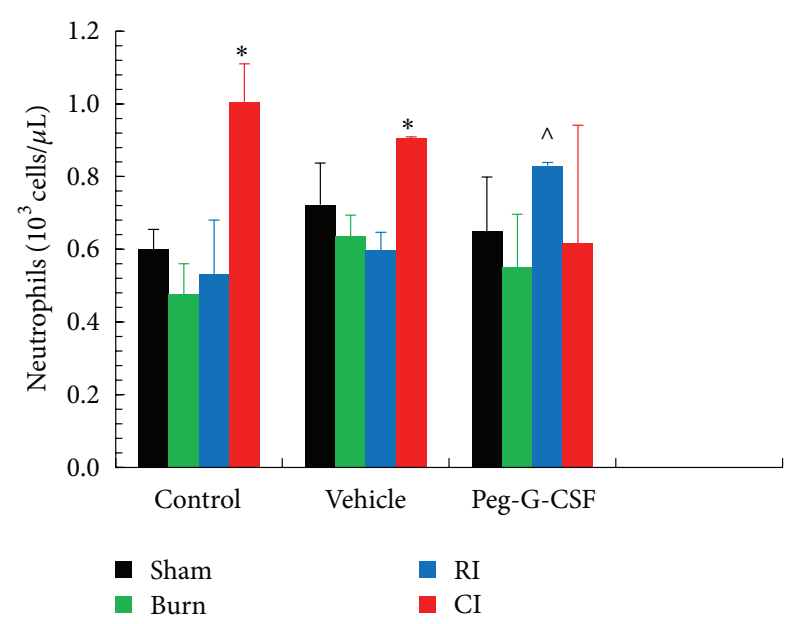

(b) Neutrophils

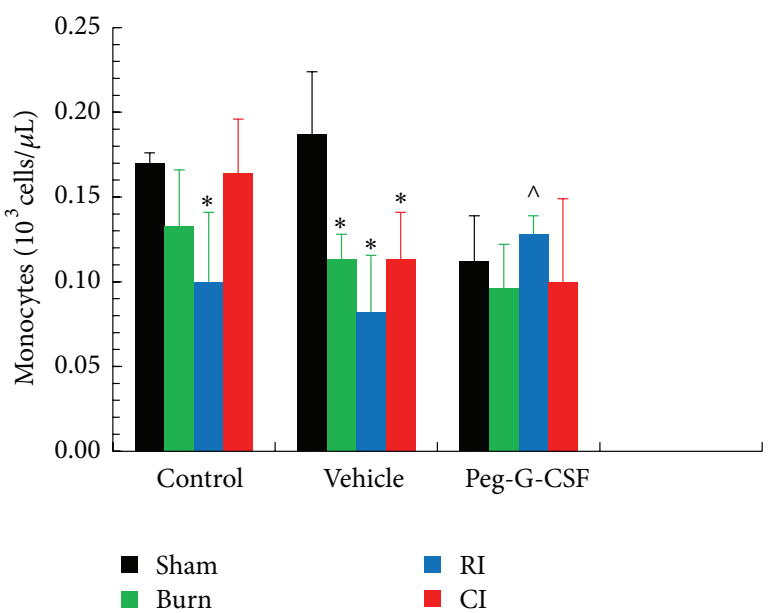

(d) Monocytes

FIGURE 4: Peg-G-CSF mitigated WBC depletion after whole-body ionizing irradiation alone but not after irradiation combined with skin burn at day 30 after burn, RI, or CI. $N=6$ per group. ${ }^{*} P<0.05$ versus sham and burn; ${ }^{\#} P<0.05$ versus RI; ${ }^{\wedge} P<0.05$ versus RI + Vehicle. RI: 9.5 Gy; CI: 9.5 Gy and skin burn.

s.c. injections of the recombinant G-CSF peptide to the same strain of irradiated mice daily for 14 days improved 30-day survival by only $25 \%$ (Figure 3 ). However, the recombinant G-CSF peptide was effective in improving CI mice survival by an incremental difference of $20 \%$ above the control (Figure 3 ). In contrast to the RI mice, peg-G-CSF failed to improve survival after CI. This could be due to the complexity of mechanisms of CI involving enhancements of serum cytokines/chemokines and systemic bacterial infection [7, 28,29 ] that requires more than peg-G-CSF to manage the imbalance of homeostasis. G-CSF was also used with IL3 to mobilize bone marrow hematopoietic progenitors to circulation [14].

$\mathrm{RI}$ and $\mathrm{CI}$ significantly reduced $\mathrm{WBC}, \mathrm{RBC}$, and platelet counts $[1,2]$. At day 30 after RI or CI, surviving mice still displayed low values for WBC, mainly neutrophils and lymphocytes (Figure 4). However, the RI-induced decreases were mitigated slightly yet significantly in peg-G-CSF-treated mice. Peg-G-CSF is known to initiate proliferation and differentiation of myeloid progenitors into mature granulocytes and induce hematopoietic stem cell mobilization from the bone marrow into the bloodstream making it effective in the recovery from infection $[11,12]$ and wound healing [13]. PegG-CSF, when combined with stem cell factor and erythropoietin, was used to treat a technician, who was exposed to gamma radiation [14]. From our study, we postulate further that peg-G-CSF mobilizes hematopoietic progenitor cells in addition to myeloid cells to peripheral blood to mitigate the blood-cell depletion (Figure 5).

Peg-G-CSF treatment improved platelet counts in surviving RI-mice but not in surviving CI-mice, suggesting that this factor also can stimulate megakaryocytes in the bone marrow, similar to platelet recovery resulting from IL-12 treatment [36].

We observed that the RI mice but not CI mice exhibited splenomegaly. Splenomegaly is usually associated with 


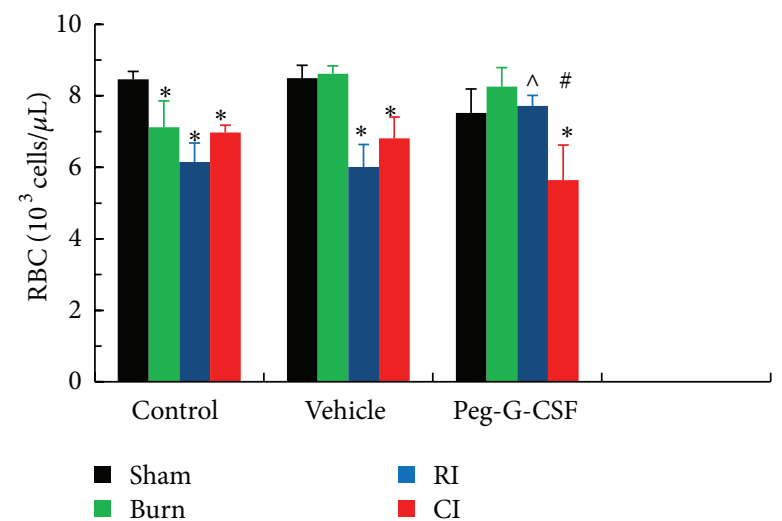

(a) $\mathrm{RBC}$

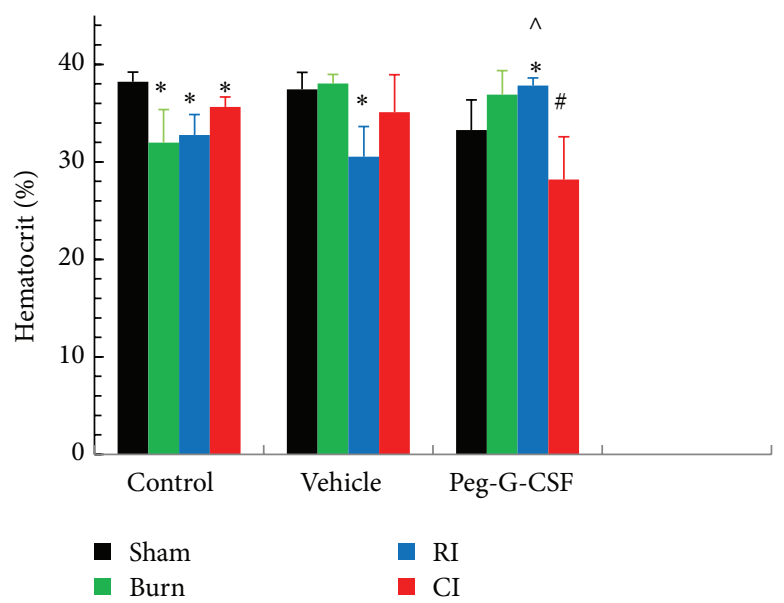

(c) Hematocrit

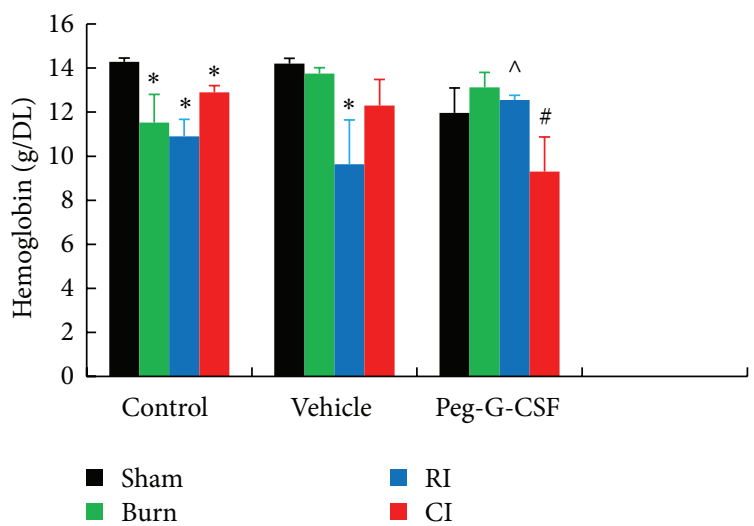

(b) Hemoglobin

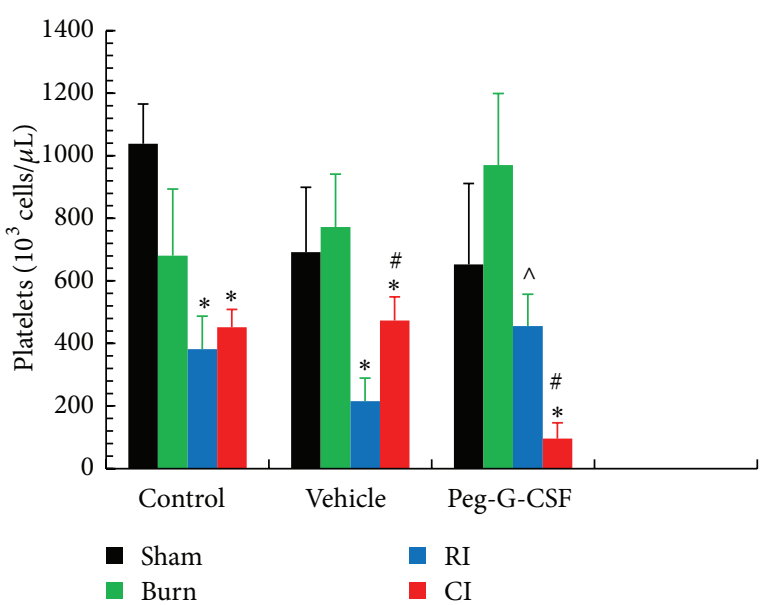

(d) Platelets

FIGURE 5: Peg-G-CSF mitigated both RBC depletion and platelet depletion after whole-body ionizing irradiation alone but not after irradiation combined with skin burn at day 30 after burn, RI, or CI. $N=6$ per group. ${ }^{*} P<0.05$ versus sham; ${ }^{\wedge} P<0.05$ versus RI + Vehicle. ${ }^{\#} P<0.05$ versus RI. RI: 9.5 Gy; CI: 9.5 Gy and skin burn.

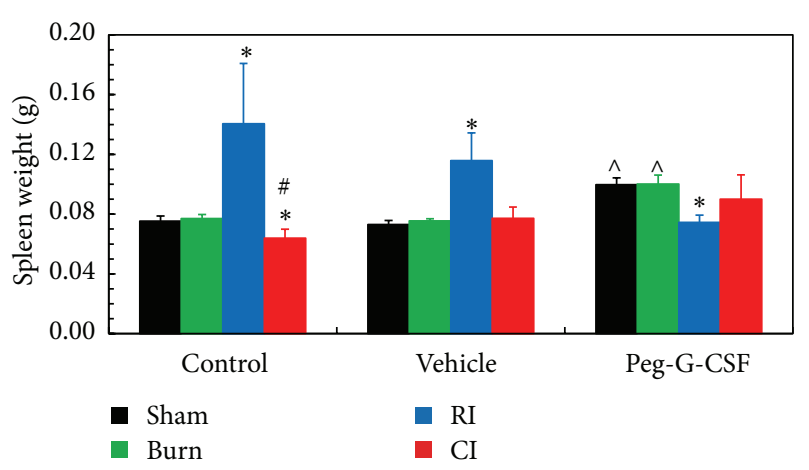

(a) Spleen weight

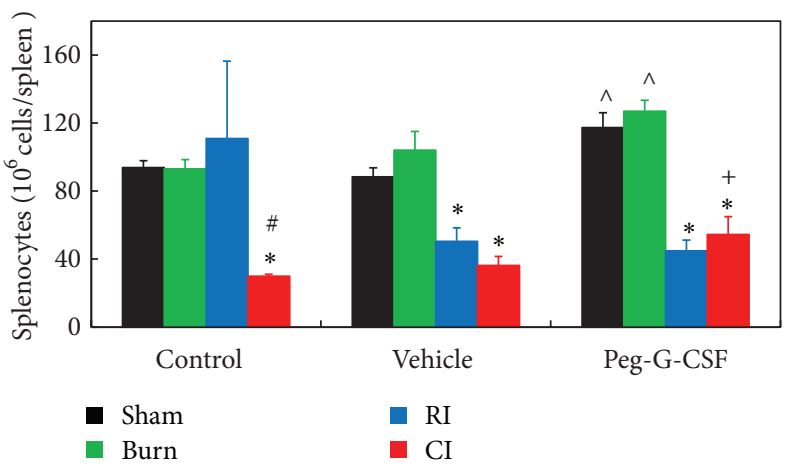

(b) Splenocytes

FIGURE 6: Peg-G-CSF mitigated both increased spleen weights and decreased splenocyte counts after whole-body ionizing irradiation alone and increased splenocyte counts after irradiation combined with skin burn at day 30 after burn, RI, or CI. $N=6$ per group. ${ }^{*} P<0.05$ versus sham and burn; ${ }^{\#} P<0.05$ versus RI; ${ }^{\wedge} P<0.05$ versus sham + Vehicle and burn + Vehicle; ${ }^{+} P<0.05$ versus CI + Vehicle. RI: 9.5 Gy; CI: $9.5 \mathrm{~Gy}$ and skin burn. 


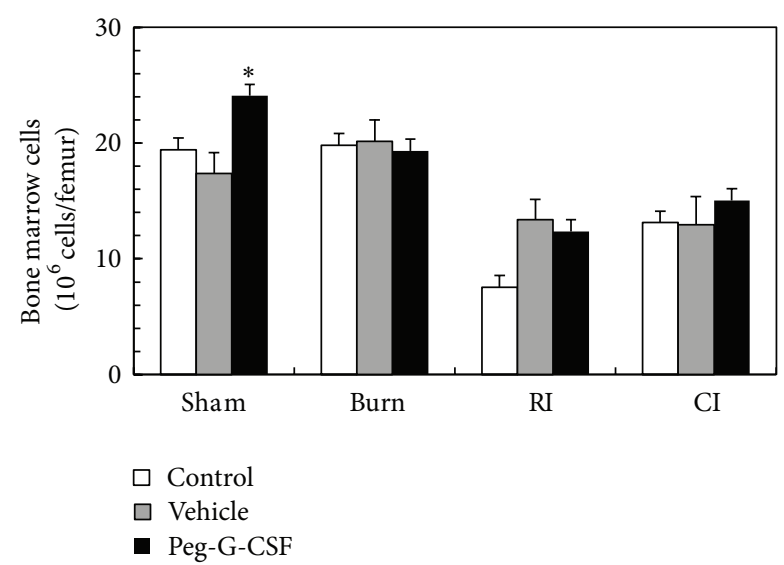

Figure 7: Peg-G-CSF increased bone marrow cell counts in nonirradiated control mice at day 30 after burn, RI, or CI. $N=6$ per group. ${ }^{*} P<0.05$ versus all other groups. RI: $9.5 \mathrm{~Gy}$; CI: 9.5 Gy and skin burn.

disease processes that involve the destruction of abnormal RBC in the spleen. From our results, we speculate that splenomegaly may be caused by removal of RBC after irradiation. Several questions are raised. For example, how did the red and the white pulp of the spleen look like and the different cell types get distributed in the spleen? Where did injured RBC get trapped in the spleen after RI? It is also unclear why the spleen weight gained in vehicle-treated mice after RI or CI but their splenocyte counts were less than that in the sham group. Perhaps, injured RBC trapped in the spleen may increase the spleen weight but release unidentified factors that can inhibit splenocyte recovery. Further studies will be needed to address these questions.

It appears that treatment with peg-G-CSF mitigated RIinduced erythropenia and anemia. This may have been due to the drug's ability to release other cytokines that can accelerate maturation of erythroid cells in bone marrow (i.e., hematopoietic erythropoiesis) and/or in spleen (i.e., stress erythropoiesis) and to mobilize them to peripheral blood [37]. Further studies in these regards to elucidate the RIinduced splenomegaly will surely be eagerly anticipated.

It is evident that $\mathrm{CI}$ enhances $\mathrm{RBC}$ depletion, hemoglobin reduction, and hematocrit declination [1] as well as more systemic bacterial infection $[4,7]$. It is not understood why CI did not induce splenomegaly but is likely associated with injury to the skin.

$\mathrm{RI}$ and CI result in systemic bacterial infection leading to magnified increases in cytokine concentrations in serum [7]. Acute bacterial inflammation is accompanied by excessive production of reactive oxygen and nitrogen species (ROS and RNS), which ultimately results in redox stress, a leading pathogenic factor of the septic multiple organ dysfunction syndromes $[38,39]$. It is reported that in an in vitro study mesenchymal stromal cells survive lipopolysaccharide challenge, partially due to adaptive responses to septic oxidative stress [40]. Therefore, the possibility of survival improvement in peg-G-CSF treated RI mice mediated by adaptive responses to septic oxidative stress cannot be excluded. Additional studies are ongoing.

In summary, skin burns increased radiation-induced mortality and body-weight loss. Peg-G-CSF treatment enhanced 30 -day survival to $100 \%$, significantly mitigated bodyweight loss, WBC depletion, RBC depletion, platelet depletion, and splenomegaly in RI mice. These results demonstrate efficacy of peg-G-CSF as a radiomitigator.

$\begin{array}{ll}\text { Abbreviations } \\ \text { AFRRI: } & \text { Armed Forces Radiobiology Research } \\ & \text { Institute } \\ \text { CI: } & \text { Combined injury } \\ \text { i.p.: } & \text { Intraperitoneal } \\ \text { p.o.: } & \text { Oral gavage } \\ \text { Peg-G-CSF: } & \text { Pegylated granulocyte colony stimulating } \\ & \text { factor } \\ \text { RBC: } & \text { Red blood cells } \\ \text { RCI: } & \text { Radiation combined injury } \\ \text { RI: } & \text { Radiation injury } \\ \text { s.c.: } & \text { Subcutaneous } \\ \text { TBSA: } & \text { Total body surface area } \\ \text { VSD: } & \text { Veterinary Science Department } \\ \text { W: } & \text { Wound } \\ \text { WBC: } & \text { White blood cells. }\end{array}$

\section{Disclaimer}

The views, opinions, and findings contained in this report are those of the authors and do not reflect official policy or positions of the Armed Forces Radiobiology Research Institute, the Uniformed Services University of the Health Sciences, the National Institute of Allergy and Infectious Diseases, the Department of Defense, or the United States government. The commercial products identified in this document do not imply recommendation or endorsement by the federal government and do not imply that the products identified are necessarily the best available for the purpose.

\section{Conflict of Interests}

The authors declare that they have no conflict of interests.

\section{Authors' Contribution}

Juliann G. Kiang conceived and designed the experiments in the paper. Juliann G. Kiang, Min Zhai, Pei-Jyun Liao, David L. Bolduc, Thomas B. Elliott, and Nikolai V. Gorbunov performed the experiments. Juliann G. Kiang, Min Zhai, and Pei-Jyun Liao analyzed data and graphed the figures. Thomas B. Elliott performed the log-rank test. Juliann G. Kiang wrote the paper. All authors read, edited, and approved the paper.

\section{Acknowledgments}

The authors gratefully acknowledge LTC Rebecca Holt and the Veterinary Sciences Department staff for animal care, 
Dr. Vitaly Nagy and Radiation Dosimetry staff for conducting whole-body irradiation, and Dr. Xinyue Lu, Dr. Dilber Nurmemet, Marsha Anderson, HM1, USN, Ms. Joan Smith, Mr. True Burns, and Dr. Risaku Fukumoto for their technical assistance. Research was supported by NIH/NIAID YI-AI5045-04 to Juliann G. Kiang and AFRRI RAB32164 to Thomas B. Elliott and Juliann G. Kiang.

\section{References}

[1] J. G. Kiang, B. R. Garrison, T. M. Burns et al., "Wound trauma alters ionizing radiation dose assessment," Cell and Bioscience, vol. 2, no. 1, article 20, 12 pages, 2012.

[2] J. G. Kiang, B. R. Garrison, R. Fukumoto, and T. B. Elliott, "Ciprofloxacin inhibits gamma radiation-induced increases in $\gamma$-H2AX, p53 phosphorylation in human tumor cells and p53 phosphorylation, Gadd45a, bax, and Bcl-2 gene expression in human peripheral blood cells," in Proceedings of the 56th Radiation Research Society Annual Meeting, p. 188, Maui, Hawaii, USA, September 2010.

[3] R. Fukumoto and J. G. Kiang, "Geldanamycin analog 17DMAG limits apoptosis in human peripheral blood cells by inhibition of p53 activation and its interaction with heat-shock protein $90 \mathrm{kDa}$ after exposure to ionizing radiation," Radiation Research, vol. 176, no. 3, pp. 333-345, 2011.

[4] R. Fukumoto, L. H. Cary, N. V. Gorbunov, T. B. Elliott, and J. G. Kiang, "Ciprofloxacin modulates cytokine profiles, accelerates bone marrow recovery and mitigates ileum injury after radiation combined with wound trauma," PLoS ONE, vol. 8, no. 3, Article ID e58389, 11 pages, 2013.

[5] G. D. Ledney and T. B. Elliott, "Combined injury: factors with potential to impact radiation dose assessments," Health Physics, vol. 98 , no. 2, pp. 145-152, 2010.

[6] J. G. Kiang and G. D. Ledney, "Skin injuries reduce survival and modulate corticosterone, C-reactive protein, complement component 3, IgM, and prostaglandin E2 after whole-body reactorproduced mixed field ( $n+\gamma$-photons) irradiation," Oxidative Medicine and Cellular Longevity, vol. 2013, Article ID 821541, 10 pages, 2013.

[7] J. G. Kiang, W. Jiao, L. H. Cary et al., "Wound trauma increases radiation-induced mortality by activation of iNOS pathway and elevation of cytokine concentrations and bacterial infection," Radiation Research, vol. 173, no. 3, pp. 319-332, 2010.

[8] M. E. Berger, D. M. Christensen, P. C. Lowry, O. W. Jones, and A. L. Wiley, "Medical management of radiation injuries: current approaches," Occupational Medicine, vol. 56, no. 3, pp. 162-172, 2006.

[9] J. K. Waselenko, T. J. MacVittie, W. F. Blakely et al., "Medical management of the acute radiation syndrome: recommendations of the strategic national stockpile radiation working group," Annals of Internal Medicine, vol. 140, no. 12, pp. 10371051, 2004.

[10] G. Molineux, "The design and development of pegfilgrastim (PEG-rmetHuG-CSF, Neulasta)," Current Pharmaceutical Design, vol. 10, no. 11, pp. 1235-1244, 2004.

[11] D. Metcalf, "The role of the colony-stimulating factors in the treatment of infections," in Frontiers of Infectious Diseases: New Antibacterial Strategies: Proceedings of an International Symposium Sponsored by Glaxo Research, Brocker Hall, Hertfordshire 30 June-3 July 1990, H. C. Neu, Ed., Churchill Livingstone, New York, NY, USA, 1990.
[12] D. Metcalf, "Hematopoietic cytokines," Blood, vol. 111, no. 2, pp. 485-491, 2008.

[13] E. V. Badiavas, M. Abedi, J. Butmarc, V. Falanga, and P. Quesenberry, "Participation of bone marrow derived cells in cutaneous wound healing," Journal of Cellular Physiology, vol. 196, no. 2, pp. 245-250, 2003.

[14] J. M. Bertho, N. M. Griffiths, and P. Gourmelon, "The medical diagnosis and treatment of radiation overexposed people," 2003, http://irpall.irpa.net/pdfs/RC-7a.pdf.

[15] C. A. Montgomery, "Oncologic and toxicologic research: alleviation and control of pain and distress in laboratory animals," Cancer Bulletin, vol. 42, pp. 230-237, 1990.

[16] S. P. Tomasivic, L. G. Coghlan, K. N. Gray, A. J. Mastromarino, and E. L. Travis, "IACUC evaluation of experiments requiring death as an end point: a cancer center's recommendations," $L a b$ Animal, pp. 31-34, 1988.

[17] International Standardization Organization and ASTM International, Standard Practice for Use of an Alanine-EPR Dosimetry System, Standard 51607:2004(E), ISO, Geneva, Switzerland; ASTM International, West Conshohocken, Pa, USA, 2004.

[18] B. I. Lord, L. B. Woolford, and G. Molineux, "Kinetics of neutrophil production in normal and neutropenic animals during the response to filgrastim ( $\mathrm{r}$-metHu G-CSF) or filgrastim SD/01 (PEG-r-metHu G-CSF)," Clinical Cancer Research, vol. 7, no. 7, pp. 2085-2090, 2001.

[19] A. B. van Spriel, I. E. van den Herik-Oudijk, and J. G. J. van de Winkel, "A single injection of polyethylene-glycol granulocyte colony-stimulating factor strongly prolongs survival of mice with systemic candidiasis," Cytokine, vol. 12, no. 6, pp. 666-670, 2000.

[20] K. J. Neelis, S. C. C. Hartong, T. Egeland, G. R. Thomas, D. L. Eaton, and G. Wagemaker, "The efficacy of single-dose administration of thrombopoietin with coadministration of either granulocyte/macrophage or granulocyte colony-stimulating factor in myelosuppressed rhesus monkeys," Blood, vol. 90, no. 7, pp. 2565-2573, 1997.

[21] E. L. Alpen and G. E. Sheline, "The combined effects of thermal burns and whole-body X-radiation on survival time and mortality," Annuls of Surgery, vol. 140, no. 1, pp. 113-118, 1954.

[22] F. A. Valeriote and D. G. Baker, "The combined effects of thermal trauma and X-irradiation on early," Radiation Research, vol. 22 , pp. 693-702, 1964

[23] B. Korlof, "Infection of burns. I. A bacteriological and clinical study of 99 cases. II. Animal experiments: burns and total body x-irradiation," Acta Chirurgica Scandinavica, Supplementum, vol. 209, pp. 1-144, 1956.

[24] J. W. Brooks, E. I. Evans, W. T. Ham Jr., and J. D. Reid, "The influence of external body radiation on mortality from thermal burns," Annals of Surgery, vol. 136, no. 3, pp. 533-545, 1952.

[25] H. Baxter, J. A. Drummond, L. G. Stephens-Newsham, and R. G. Randall, "Studies on acute total body irradiation in animals. I. Effect of streptomycin following exposure to a thermal burn and irradiation," Plastic Reconstruction Surgery, vol. 12, no. 6, pp. 439-445, 1953.

[26] G. D. Ledney, T. B. Elliott, and M. M. Moore, "Modulations of mortality by tissue trauma and sepsis in mice after radiation injury," in The Biological Basis of Radiation Protection Practice, K. L. Mossman and W. A. Mills, Eds., pp. 202-217, Williams \& Wilkins, Baltimore, Md, USA, 1992.

[27] G. D. Ledney, D. A. Stewart, E. D. Exum, and P. A. Sheehy, "Skin wound-enhanced survival and myelocytopoiesis in mice 
after whole-body irradiation," Acta Radiologica: Oncology, vol. 20, no. 1, pp. 29-38, 1981.

[28] J. L. Palmer, C. R. Deburghgraeve, M. D. Bird, M. Hauer-Jensen, and E. J. Kovacs, "Development of a combined radiation and burn injury model," Journal of Burn Care and Research, vol. 32, no. 2, pp. 317-323, 2011.

[29] A. Jacob, K. G. Shah, R. Wu, and P. Wang, "Ghrelin as a novel therapy for radiation combined injury," Molecular Medicine, vol. 16, no. 3-4, pp. 137-143, 2010.

[30] Z. Zou, H. Sun, Y. Su, T. Cheng, and C. Luo, "Progress in research on radiation combined injury in China," Radiation Research, vol. 169, no. 6, pp. 722-729, 2008.

[31] K. L. Koenig, R. E. Goans, R. J. Hatchett et al., "Medical treatment of radiological casualties: current concepts," Annals of Emergency Medicine, vol. 45, no. 6, pp. 643-652, 2005.

[32] Z. Lausevic, M. Lausevic, J. Trbojevic-Stankovic, S. Krstic, and B. Stojimirovic, "Predicting multiple organ failure in patients with severe trauma," Canadian Journal of Surgery, vol. 51, no. 2, pp. 97-102, 2008.

[33] T. J. MacVittie, A. M. Farese, and W. Jackson III, "Defining the full therapeutic potential of recombinant growth factors in the post radiation-accident environment: the effect of supportive care plus administration of G-CSF," Health Physics, vol. 89, no. 5, pp. 546-555, 2005.

[34] V. K. Singh, O. O. Fatanmi, P. K. Singh, and M. H. Whitnall, "Role of radiation-induced granulocyte colony-stimulating factor in recovery from whole body gamma-irradiation," Cytokine, vol. 58, no. 3, pp. 406-414, 2012.

[35] M. Scholz, M. Ackermann, C. Engel, F. Emmrich, M. Loeffler, and M. Kamprad, "A pharmacokinetic model of filgrastim and pegfilgrastim application in normal mice and those with cyclophosphamide-induced granulocytopaenia," Cell Proliferation, vol. 42, no. 6, pp. 813-822, 2009.

[36] T. Chen, K. A. Burke, Y. Zhan, X. Wang, D. Shibata, and Y. Zhao, "IL-12 facilitates both the recovery of endogenous hematopoiesis and the engraftment of stem cells after ionizing radiation," Experimental Hematology, vol. 35, no. 2, pp. 203-213, 2007.

[37] S. Millot, V. Andrieu, P. Letteron et al., "Erythropoietin stimulates spleen BMP4-dependent stress erythropoiesis and partially corrects anemia in a mouse model of generalized inflammation," Blood, vol. 116, no. 26, pp. 6072-6081, 2010.

[38] J. A. Russell, "Management of sepsis," The New England Journal of Medicine, vol. 355, no. 16, pp. 1699-1713, 2006.

[39] M. Perl, C. Chung, R. Swan, and A. Ayala, "Role of programmed cell death in the immunopathogenesis of sepsis," Drug Discovery Today, vol. 4, no. 4, pp. 223-230, 2007.

[40] N. V. Gorbunov, B. R. Garrison, D. P. McDaniel et al., "Adaptive redox response of mesenchymal stromal cells to stimulation with lipopolysaccharide inflammagen: mechanisms of remodeling of tissue barriers in sepsis," Oxidative Medicine and Cellular Longevity, vol. 2013, Article ID 186795, 16 pages, 2013. 


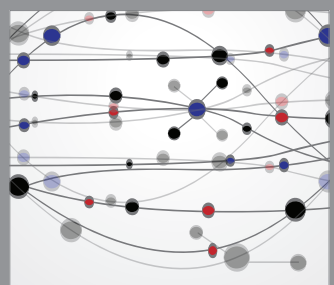

The Scientific World Journal
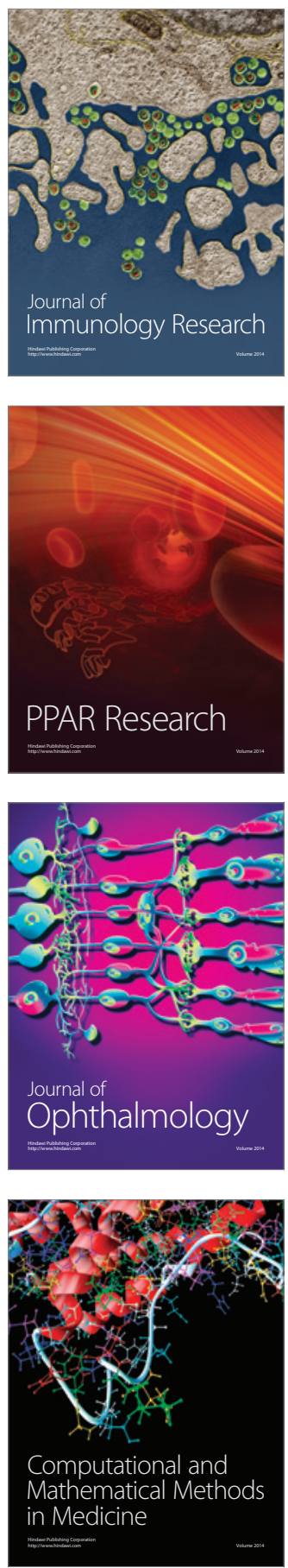

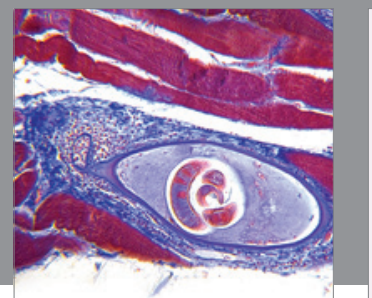

Gastroenterology

Research and Practice
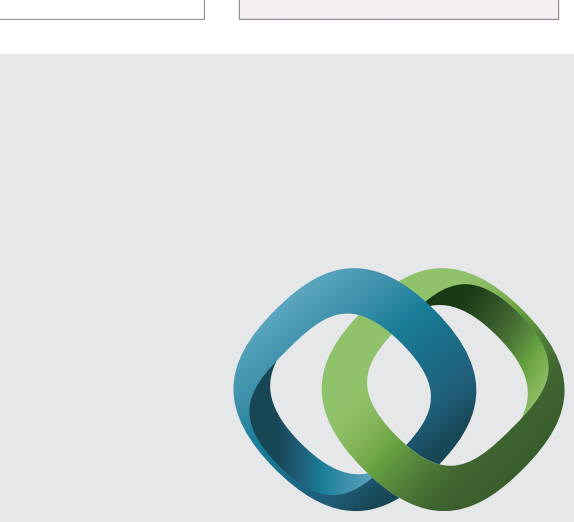

\section{Hindawi}

Submit your manuscripts at

http://www.hindawi.com
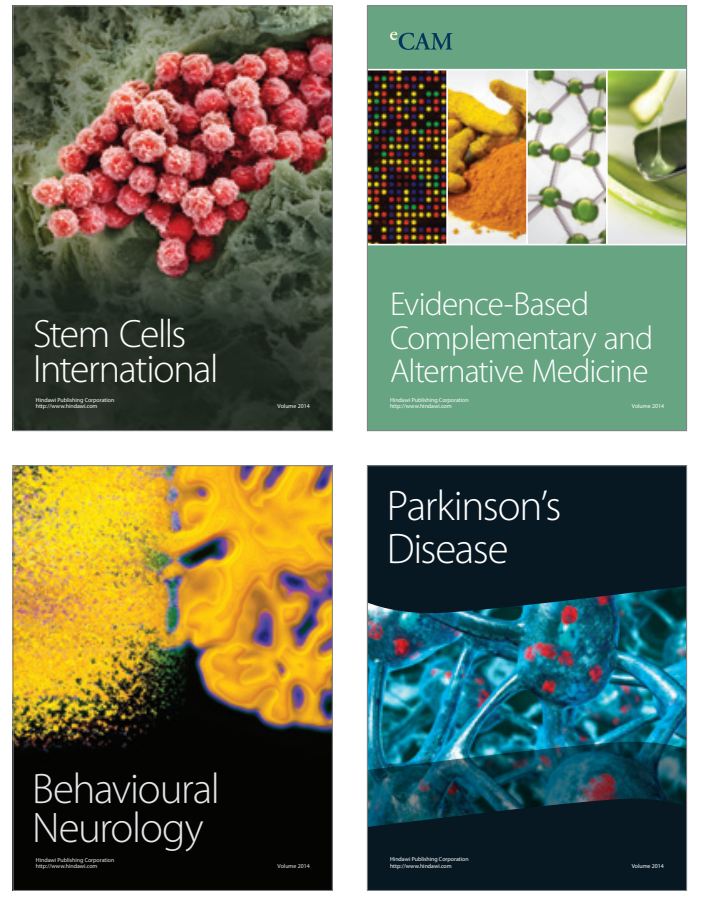
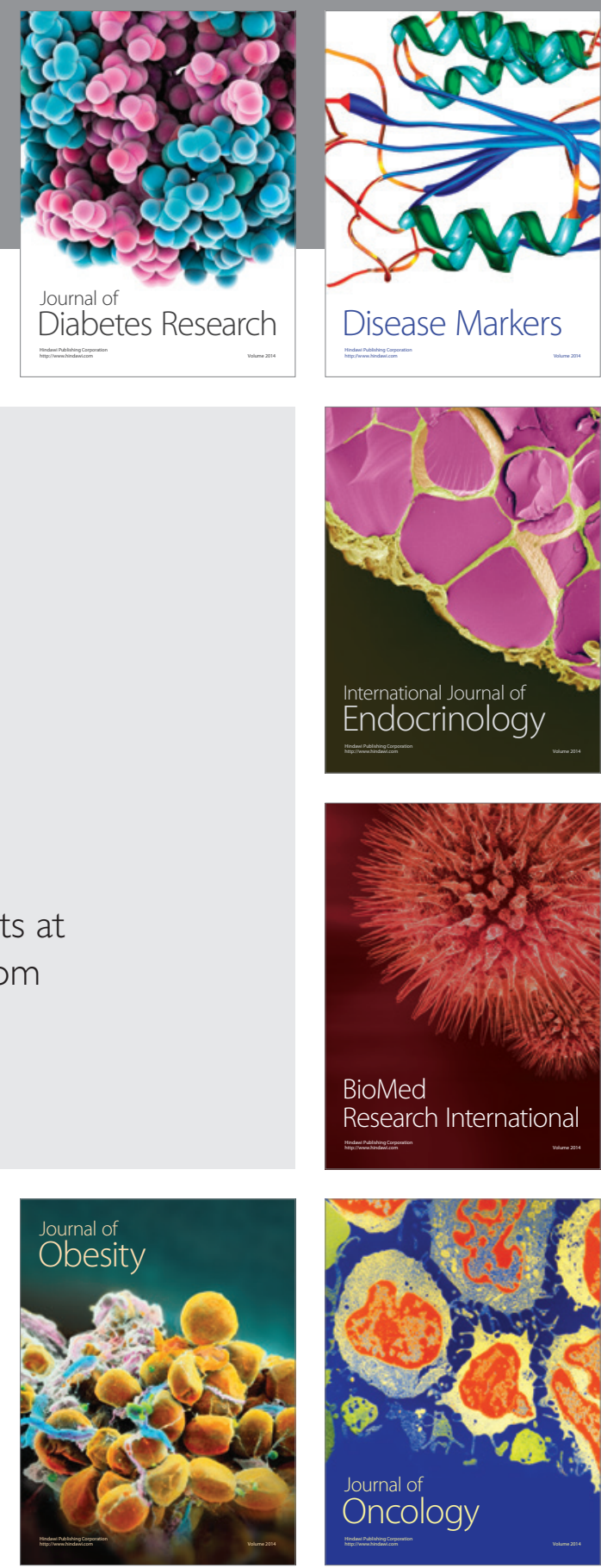

Disease Markers
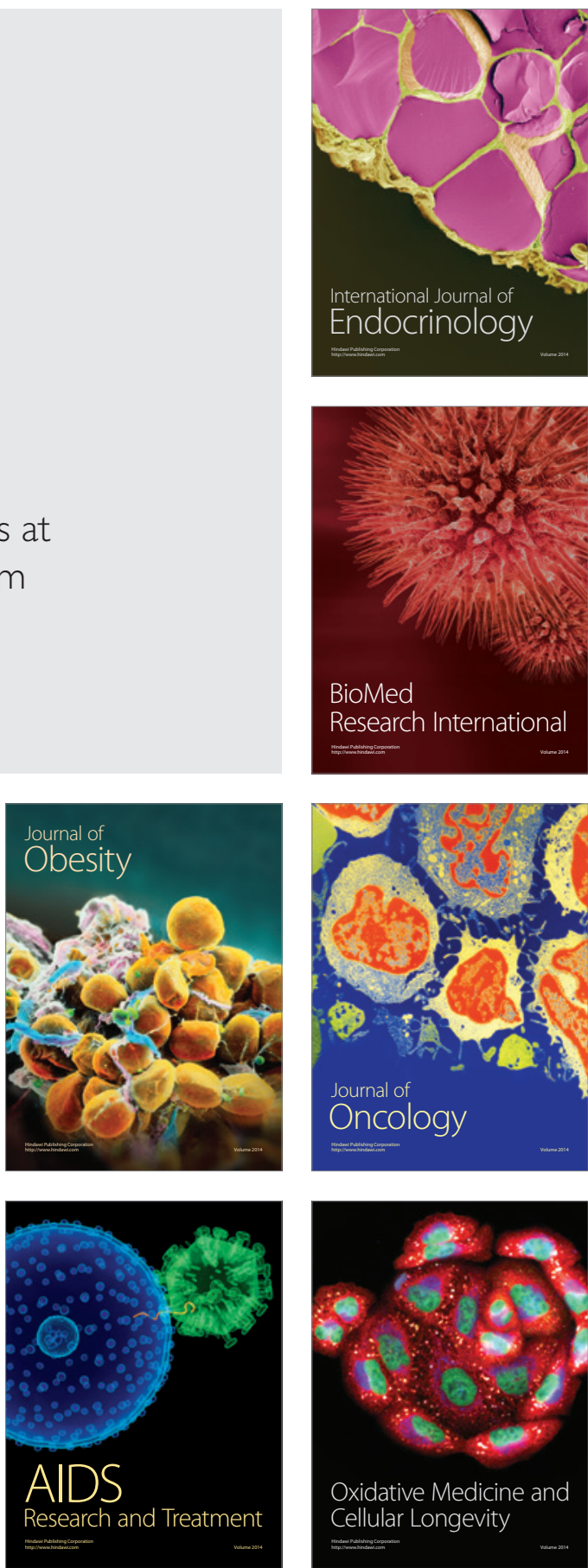